\title{
Associação entre Força Muscular Relativa e Indicadores de Obesidade em Professores Universitários
}

\author{
ASSOCIATION BETWEEN RELATIVE MUSCLE STRENGTH AND INDICATORS OF OBESITY IN UNIVERSITY PROFESSORS
}

Sandra Aires Ferreira', Denilson de Castro Teixeira', Timothy Gustavo Cavazzotto², Mayla Fernanda de Moura Cavalhaes ${ }^{3}$, Denis

Antônio Figueredo Diesel ${ }^{3}$, William Cordeiro de Souza ${ }^{4}$, Marcos Roberto Queiroga ${ }^{2}$

1. Universidade Estadual de Londrina (Uel), Londrina, PR, Brasil.

2. Universidade Estadual do Centro-Oeste (Unicentro), Guarapuava, PR, Brasil.

3. Núcleo de Estudos em Atividade Fisica, Universidade do Contestado (UnC), Porto União, SC, Brasil.

\section{RESUMO}

0 objetivo do estudo foi verificar a associação entre a força muscular relativa e os indicadores de obesidade em professores universitários. 71 docentes (37 homens e 34 mulheres) foram submetidos à medida da circunferência da cintura (CC) e ao teste de preensão manual. A partir da massa corporal (MC) e da estatura calculou-se o Índice de Massa Corporal (IMC). A força muscular relativa (\% FM) foi determinada mediante razão dos valores da média de preensão manual direita e esquerda pela MC. Com base nos valores de \% FM a amostra foi estratificada em tercis de acordo com o gênero para efeito de comparação da MC, IMC e CC utilizando o teste de Kruskal Wallis. Para identificar a diferença entre dois grupos utilizou-se a MannWhitney e para correlação usou-se o coeficiente de Spearman. Os homens do $3^{\circ}$ tercil apresentaram valores significativamente menores nos indicadores de obesidade quando comparados ao $1^{\circ}$ e $2^{\circ}$ tercil. Não houve diferença significativa para as mulheres nos indicadores de obesidade embora os valores encontrados tenham sido menores com o aumento do nível de força. Foram observadas correlações inversas para MC, IMC e CC em ambos os gêneros. 0 desempenho de força muscular palmar sugere maior importância na prevenção de obesidade entre os professores do que entre as professoras.

(Ferreira S, Teixeira D, Cavazzotto T, Cavalhaes M, Figueredo D, Cordeiro de Souza W, Queiroga M, 2018. Associação entre Força Muscular Relativa e Indicadores de Obesidade em Professores Universitários. Cienc Trab. Ene-Abr; 20 [61]: 31-35).

Palavras-chave: ATIVIDADE FÍSICA; DOCENTES; SAÚDE OCUPACIONAL.

\section{ABSTRACT}

The aim of the study was to verify the association between relative muscular strength and indicators of obesity in university professors. 71 teachers ( 37 men and 34 women) were submitted to measurement of waist circumference (WC) and the handgrip test. Based on body mass (BM) and height was calculated Body Mass Index (BMI). Relative muscle strength (\% FM) was determined by ratio of the averaged values of right and left handgrip by MC. Based on the values of the \% FM, the sample was stratified into tertiles according to gender for comparison of MC, BMI and WC using the Kruskal-Wallis test; to identify the difference between two groups was used the MannWhitney test and for correlation was used the Spearman coefficient. Men of the 3rd tertile showed lower values significantly on indicators of obesity when compared to the 1st and 2nd tertile. There was no difference for women in obesity indicators, although the values found were lower with increasing level strength. The inverse correlations were observed for MC, BMI and WC in both genders. The performance of strength palmar prehension suggests higher importance in the prevention of obesity between men than women professors.

Key words: PHYSICAL ACTIVITY; TEACHERS; OCCUPATIONAL HEALTH.

\section{INTRODUÇÃO}

Caracterizada pelo acúmulo excessivo de gordura corporal, a obesidade é considerada uma doença crônica ${ }^{1}$ e está associada a

\author{
Correspondencia / Correspondence: \\ Marcos Roberto Queiroga \\ Universidade Estadual do Centro-Oeste, Departamento de Educação Física \\ Rua Simeão Camargo Varela de Sá, no 3, Bairro Cascavel, \\ Guarapuava-PR, Brasil. \\ CEP: 85040-080. \\ e-mail:queirogamr@hotmail.com
}

Recibido: 15 de Septiembre de 2017 / Aceptado: 23 de Enero de 2018 aspectos fisiológicos, comportamentais e ao estilo de vida. ${ }^{2}$ Sua prevalência nos países desenvolvidos e em desenvolvimento vem aumentando a cada década. ${ }^{3}$ No Brasil, estima-se que 53,8\% da população apresenta sobrepeso, dos quais 18,9\% já possuem obesidade. $^{4}$

A atividade física é recomendada como um tratamento não medicamentoso para minimizar os riscos que o excesso de peso provoca a saúde de indivíduos de diferentes faixas-etárias. ${ }^{5}$ A combinação de exercícios aeróbicos e anaeróbicos demonstra impactos positivos no controle e tratamento da obesidade mediante aumento da aptidão física geral. ${ }^{6,7} 0$ treinamento resistido tem se destacado entre as recomendações para a promoção da saúde. ${ }^{8}$ Há evidências que o aumento da força muscular produz benefícios relacionados à redução da adiposidade, aumento da densidade mineral óssea e melhora da saúde cognitiva., ${ }^{9,10}$ 
Parte da redução da demanda energética observada nos últimos anos se deve ao menor envolvimento das pessoas com atividades físicas que exigem a força muscular, como as atividades da vida diária (AVD) (atividades domésticas, transporte, lazer ou trabalho). A atividade laboral do professor universitário, em função das recentes necessidades de produção científica aliada a outras tarefas, em muitos casos extrapola a carga horária de trabalho. Estas tarefas específicas contribuem para transformar a ocupação do docente universitário como uma atividade de baixa demanda energética. ${ }^{11}$ Alguns estudos já demonstraram aumento na incidência de obesidade em professores o que pode interferir diretamente na qualidade de vida e no próprio trabalho. ${ }^{12-14}$ Diante do exposto o objetivo do estudo foi verificar a associação entre a força muscular relativa e os indicadores de obesidade em professores universitários.

\section{MÉTODOS}

O Campus CEDETEG da Universidade Estadual do Centro-Oeste (Guarapuava-PR) possui 251 docentes entre efetivos e colaboradores. Uma lista com os nomes dos professores de todos os departamentos pedagógicos do campus $(\mathrm{n}=12)$ foi fornecida pela Pró-Reitora de Recursos Humanos da instituição. Desta relação, foram selecionados aleatoriamente 80 professores (30\%) dos quais 71 completaram todos os requesitos (37 homens e 34 mulheres). As informações para o estudo foram coletadas em dia de reunião departamental. Foram excluídos e substituídos pelos suplentes pré-selecionados os docentes em afastamento bem como aqueles que não estavam presentes no dia da coleta.

Todos os participantes foram previamente informados em relação aos procedimentos aos quais seriam submetidos. Em seguida, assinaram um termo de consentimento livre e esclarecido. A pesquisa foi analisada e aprovada pelo Comitê de Ética em Pesquisa da Universidade Estadual do Centro-Oeste (UNICENTR0) conforme folha de rosto no 416722 e parecer no 047/2011.

Após a seleção, os participantes responderam a um questionário de identificação. As medidas de massa corporal (MC) e estatura foram auto-referidas pelos participantes. A seguir, a circunferência da cintura (CC) foi mensurada em duplicata no ponto médio entre as últimas costelas e a crista ilíaca com auxílio de uma fita métrica inextensível (Mabis ${ }^{\circledR}$, modelo Gulik, Japan). A partir dos valores de MC e estatura, foi calculado o Índice de Massa Corporal (IMC). ${ }^{15}$ A força muscular de preensão manual direita e esquerda foi determinada com dinamômetro manual (Crown ${ }^{\circledR}$, Filizola, São Paulo, Brasil) com capacidade de $100 \mathrm{kgf}$. Antes dos testes, todos os participantes foram orientados sobre o funcionamento do equipamento e dos procedimentos para realização do protocolo de medida. Também foram oferecidas duas tentativas com aplicação de força de contração manual para familiarização. Para realização do teste, os avaliados foram orientados a permaneceram em pé, com os braços estendidos ao longo do corpo, o antebraço em rotação neutra e a utilizar as últimas quatro falanges distais para exercer a força na barra de tração. A partir dessa posição, aguardaram o sinal de “preparar, já!” e executaram uma contração máxima. Em seguida o dinamômetro foi empunhado na outra mão, repetindo o mesmo procedimento. 0 teste foi realizado duas vezes em cada uma das mãos e o valor médio foi empregado para análise. A força muscular relativa (\% FM) foi estimada por meio da média dos valores obtidos pelo teste de preensão manual da mão direita e esquerda $(\mathrm{kg})$, dividido pela massa corporal $(\mathrm{kg}){ }^{16}$

A análise estatística foi realizada a partir do programa SPSS versão 15.0 para Windows, com nível de significância de $p<0,05$. 0 teste de Shapiro Wilk revelou assimetria na distribuição dos dados, por isso, foram apresentados descritivamente empregando a mediana e variância interquartil. Com base nos valores de \% FM a amostra foi estratificada em tercis ( $1^{\circ}$ tercil apresenta os individuos com menores níveis de força muscular, o $2^{\circ}$ tercil com níveis medianos e o $3^{\circ}$ tercil com os maiores níveis) para efeito de comparação da MC, IMC e CC, por meio do teste de Kruskal Wallis. Em seguida para identificar a diferença entre dois grupos foi aplicado o teste de Mann-Whitney. Para determinar a associação entre \% FM e os indicadores de obesidade empregou-se o coeficiente de correlação de Spearman.

\section{RESULTADOS}

A tabela 1 exibe as características antropométricas e os valores de desempenho de força muscular dos participantes do sexo masculino e feminino. Não houve diferença significativa para idade e estatura entre os três grupos de professores. Contudo, os docentes do sexo masculino classificados no $3^{\circ}$ tercil demonstraram menores valores para MC, IMC e CC quando comparados aos docentes incluídos no $1^{\circ}$ e $2^{\circ}$ tercil. Para as professoras houve diferença significativa apenas para a MC e a estatura entre o $1^{\circ}$ e o $2^{\circ}$ tercil.

Tabela 1.

Característica dos docentes universitários estratificados em tercis.

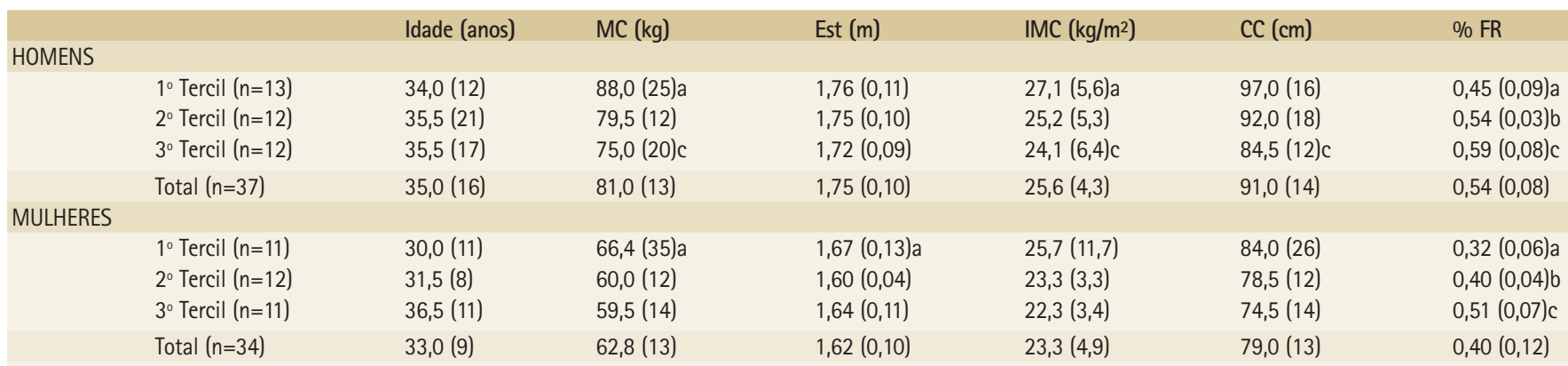

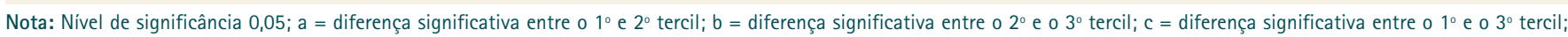

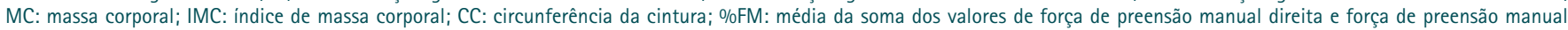
esquerda / massa corporal.

Fonte: Dados da Pesquisa, 2017. 
Figura 1.

Correlação entre força muscular relativa e indicadores de obesidade (a: MC; b: IMC; c: CC) para o sexo masculino.
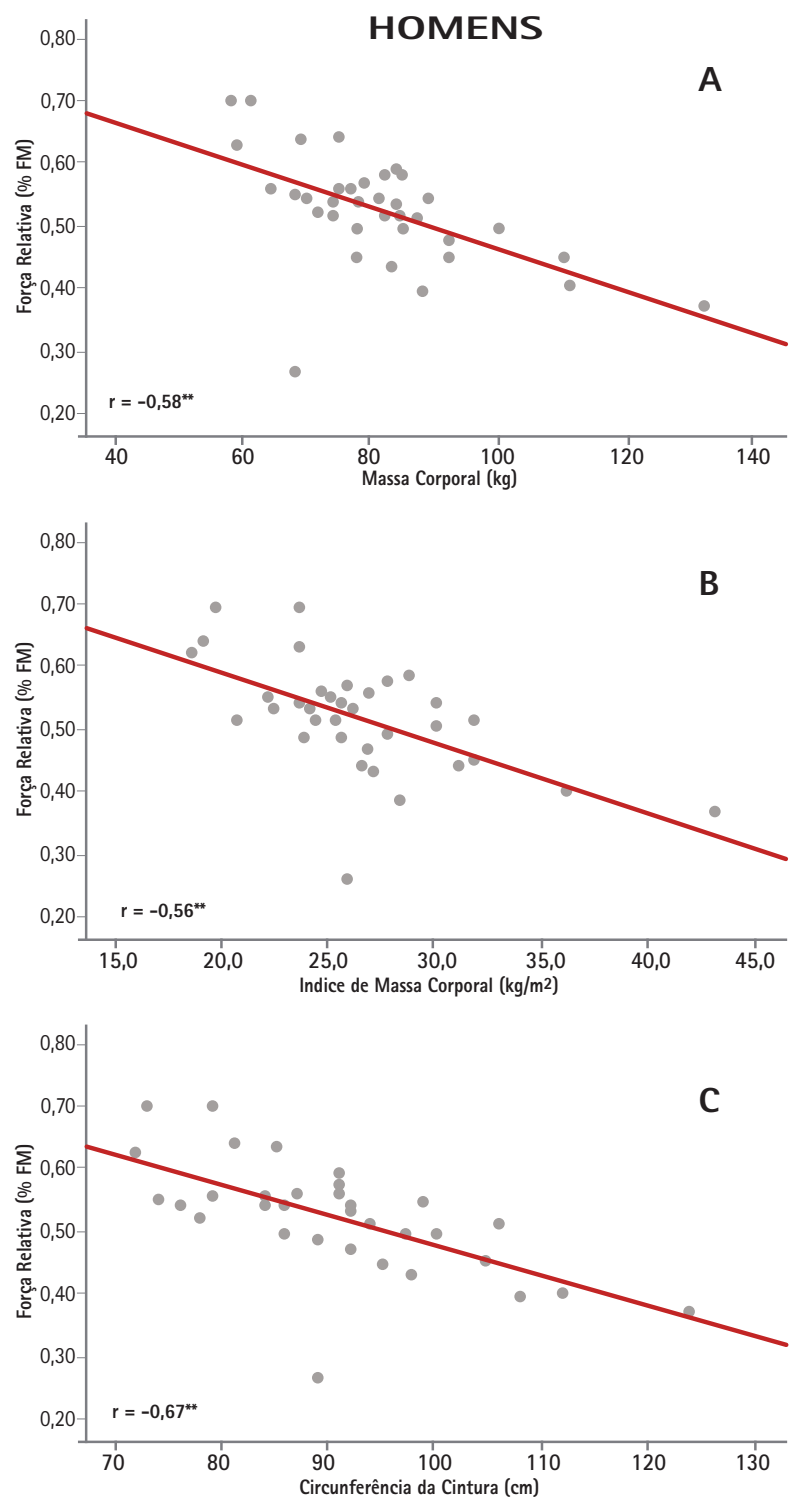

${ }^{*} p<0,05{ }^{* *} p<0,01 \quad$ Fonte: Dados da Pesquisa, 2017.

As Figuras 1 e 2 apresentam os valores de correlação entre \%FM e indicadores de obesidade (IMC e CC) para o sexo masculino ( $\mathrm{r}=$ $-0,58$ a $-0,67)$ e feminino $(r=-0,44$ a $-0,55)$, respectivamente. As informações indicam uma moderada correlação inversa entre \% FM e a MC, IMC e a CC para ambos os gêneros.

\section{DISCUSSÃO}

Os resultados do presente estudo demonstram que os homens com maior \% FM (3० tercil) apresentaram valores significativamente inferiores de MC, IMC e CC quando comparados ao grupo com menor \% FM ( $1^{\circ}$ tercil). Por sua vez, não houve diferença significativa para os indicadores de obesidade entre as professoras, inde-
Figura 2.

Correlação da força muscular relativa e indicadores de obesidade (a: $\mathrm{MC}$; b: IMC; c: CC) para o sexo feminino.
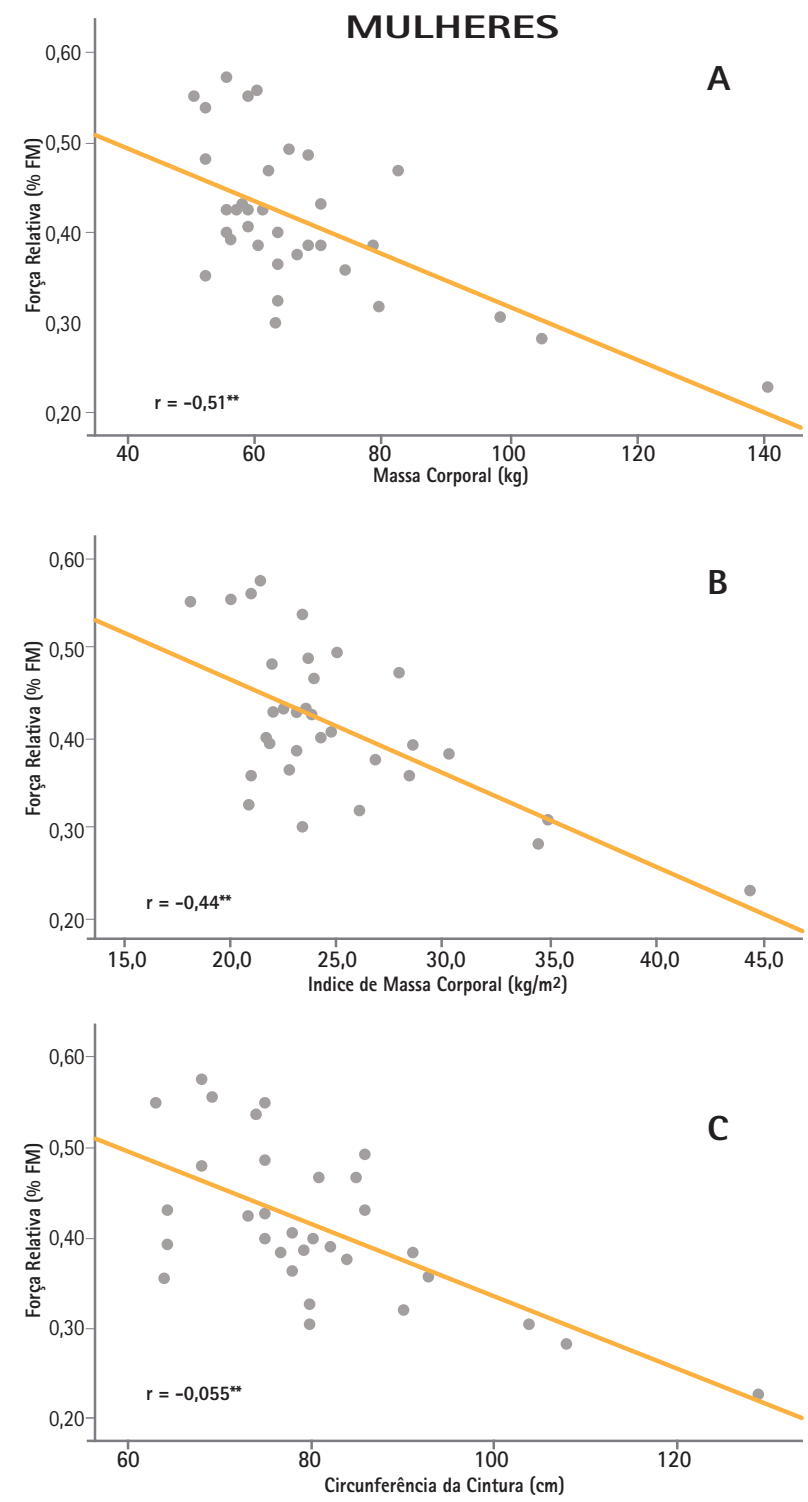

${ }^{*} p<0,05{ }^{* *} p<0,01 \quad$ Fonte: Dados da Pesquisa, 2017

pendente dos níveis de \%FM. Destaca-se que tanto a CC quanto o IMC são adotados frequentemente como indicadores de adiposidade e, representam, indiretamente, os depósitos de gordura abdominal e a gordura corporal total, respectivamente. ${ }^{17,18}$

Estudos têm evidenciado que os baixos níveis de força muscular estão fortemente associados com a morbidade e mortalidade. ${ }^{19,20}$ Considerando que a obesidade é uma enfermidade crônica, tais achados corroboram com os resultados obtidos por esse estudo, onde os indivíduos com menores niveis de força demonstraram maiores valores para os indicadores de obesidade.

0 pico de força ocorre por volta dos 30 anos e decresce de forma gradativa a partir da quinta década de vida. ${ }^{21}$ A literatura aponta que os maiores índices de obesidade são mais comuns entre os 45 e 64 anos de idade para ambos os sexos. ${ }^{22}$ Essas informações 
sugerem que a redução da força muscular e o aumento da obesidade coincidem com o mesmo período de vida. Adicionalmente, a perda da massa muscular, decorrente do envelhecimento, é acompanhada de redução da taxa metabólica o que pode contribuir para o aumento da obesidade. ${ }^{23}$ No presente estudo, foi identificado que os professores com maiores índices de força demonstraram menores indicadores de obesidade.

A associação entre força muscular e obesidade apresenta algumas controvérsias na literatura. Neste sentido, foi observado que mulheres obesas demonstraram menores valores de força muscular quando comparadas as não obesas ${ }^{24}$, porém maiores níveis de força foram relatados em mulheres com sobrepeso quando comparadas com mulheres magras. ${ }^{25} \mathrm{Em}$ nosso estudo, embora não sejam diferentes significativamente, as professoras compreendidas no $1^{\circ}$ e $2^{\circ}$ tercil demonstraram maiores valores para os indicadores de obesidade do que as professoras no $3^{\circ}$ tercil. Isto leva a crer que a associação entre a força muscular e a MC ainda não está bem esclarecida. ${ }^{26}$

Foi observada baixa a moderada associação inversa $(-0,44$ a -0,67) entre a \% FM com MC, IMC e CC para os homens (Figura 1) e para as mulheres (Figura 2). Um estudo longitudinal realizado no Canadá com 4.900 indivíduos evidenciou correlação inversa para indicadores de obesidade e força muscular em homens e mulheres. Os resultados demonstraram aumento da MC e uma diminuição gradativa da força no decorrer de 20 anos. ${ }^{27}$ Associação negativa foi observada entre \% FM, níveis pressóricos e obesidade em mulheres. ${ }^{24}$ Entre os homens foram verificados resultados semelhantes no qual maiores níveis de força apresentaram relação negativa com obesidade e hipertensão. ${ }^{28}$ Esses achados corroboram com os desta pesquisa e reforçam que a força muscular pode ser um indicador indireto de obesidade entre professores.

Uma limitação do estudo pode estar relacionada à utilização do teste de força isométrica para traduzir a força muscular geral do indivíduo, uma vez que cada grupo muscular apresenta suas particularidades (ângulo articular e tipo de contração). ${ }^{29}$ Contudo, alguns autores apontam a utilização da preensão manual como uma das principais formas de indicar a força total do corpo. ${ }^{30,31}$ Outras possíveis limitações podem ser apontadas no auto relato da massa corporal e estatura. Contudo, evidências demonstram forte concordância entre as medidas mensuradas e auto-referidas de massa corporal (MC) e estatura, especialmente em adultos de nível educacional elevado, como foi o caso de amostra do presente estudo. ${ }^{17,32}$

\section{CONCLUSÃO}

0 presente estudo demonstrou que há uma associação negativa entra a força muscular relativa e os indicadores de obesidade na amostra investigada. Para os professores, o desempenho de força apresentou impacto significativo nos indicadores de obesidade, porém isso não foi observado entre as professoras. 
1. Milic $S$, Lulic D, Štimac D. Non-alcoholic fatty liver disease and obesity: Biochemical, metabolic and clinical presentations. World J Gastroenterol. 2014; 20(28):9330-9337.

2. Dias IBF, Montenegro RA, Monteiro WD. Exercícios físicos como estratégia de prevenção e tratamento da obesidade: aspectos fisiológicos e metodológicos. Revista HUPE. 2014; 13(1):70-79.

3. Ferreira RAB, Benicio MHDA. Obesidade em mulheres brasileiras: associação com paridade e nível socioeconômico. Rev Panam Salud Pública. 2015; 37(45):337-342.4. Souza WC, Queiroga MR, Costa A, Smolarek AC, Mascarenhas LPG. Sobrepeso e obesidade em uma escola do município de Três Barras-SC. Corpoconsciência. 2017; 21(3):1-6.

5. Paes ST, Bianchini RM. Obesity: How can Interventions Ensure Treatment Success? Int J Endocrinol Metab Disord. 2015; 1(4):1-2.

6. Paes ST, Marins JCB, Adreazzi AE. Efeitos metabólicos do exercício físico na obesidade infantil: uma visão atual. Rev Paul Pediatr. 2015; 33(1):122-129.

7. Gow ML, Doornc NV, Broderick CR, Hardy LL, Ho M, Baur LA. et al. Sustained improvements in fitness and exercise tolerance in obese adolescents after a 12 week exercise intervention. Obes Res Clin Pract. 2016; 10(2):178-188.

8. Teixeira CVLS, Gomes RJ. Treinamento resistido manual e sua aplicação na educação física. Rev Bras Fisiol Exerc. 2016; 15(1):23-35.

9. Coelho Netto JS, Aptekmann NP. Efeitos do treinamento funcional sobre a composição corporal: um estudo em alunos fisicamente ativos de academia. Rev Bras Fisiol Exerc. 2016; 15(2):68-76.

10. Fleck SJ, Kraemer WJ. Fundamentos do treinamento de força muscular. 4a ed. Porto Alegre: Artmed; 2017.

11. Souza MC, Guimarães ACA, Marinho A, Matias TS Araújo CCR, Parcias SR. et al. Atividade física relacionada ao estresse no trabalho de professores universitários. R Bras Ci e Mov. 2014; 22(4):68-76.

12. He $L$, Ren $X$, Qian $Y$, Jin $Y$, Chen $Y$, Guo D. et al. Prevalence of overweight and obesity among a university faculty and staffs from 2004 to 2010, China. Nutr Hosp. 2014; 29(5):1033-1037.

13. Moreira OC, Oliveira RAR, Oliveira CEP, Doimo LA, Amorim PRS, Laterza MC. et al. Risk factors for cardiovascular disease in professors from a public university. Invest Educ Enferm. 2014; 32(2):280-290.

14. Moreira OS, Oliveira RAR, Oliveira CEP, Doimo LA, Amorin PRS, Marins JCB. Anthropometric, cardiovascular and functional variables as indicators of health related physical fitness in university professors. Fisioter Mov. 2015; 28(3):545-554.

15. Petroski EL. Antropometria: Técnicas e Padronizações. 5a ed. Fontoura: Várzea Paulista; 2011.

16. Mathiowetz V. Effects of three trials on grip and pinch strength measurements. J Hand Ther. 1990; 3(4):195-198.

17. Neto GAM, Polito MD, Lira VA. Fidedignidade entre peso e estatura reportados e medidos e a influência do histórico de atividade física em indivíduos que procuram a prática supervisionada de exercícios. Rev Bras Med Esporte. 2005; 11(2):141-145.

18. Araújo AJS, Santos ACO, Prado WL. Body composition of obese adolescents: association between adiposity indicators and cardiometabolic risk factors. J Hum Nutr Diet. 2017; 30(2):193-202.

19. Evans WJ. Sarcopenia should reflect the contribution of age-associated changes in skeletal muscle to risk of morbidity and mortality in elderly people. J Am Med Dir Assoc. 2015; 16(7):546-547.

20. Volaklis KA, Halle $M$, Meisinger $C$. Muscular strength as a strong predictor of mortality: A narrative review. Eur J Intern Med. 2015; 26(5):303-310.

21. Fitzgerald SJ, Barlow CE, Kampert JB, Morrow JRJr, Jackson AW, Blair SN. Muscular fitness and all-cause mortality: prospective observations. J Phys Act Health. 2004; 1(1):7-18.

22. Deschenes MR. Effects of aging on muscle fibre type and size. Sports Med. 2004; 34(12):809-824.

23. Falsarella GR, Gasparotto LPR, Coimbra IB, Coimbra AMV. Envelhecimento e os fenótipos da Composição Corporal. Rev Kairós Gerontol. 2014; 17(2):5777.

24. Teixeira, TG, Tibana RA, Vieira DCL, Tajra V, Balsamo S, Asano RY. et al. Comparação da força muscular entre mulheres de meia idade com e sem sobrepeso/obesidade. R bras Ci e Mov. 2014; 22(1):5-11.

25. Jurimae T, Jurimae J. Anthropometric and health-related fitness characteristics in middle-aged obese women. Coll Antropol. 1998; 22(1):97-106.

26. Martinez BP, Ramos IR, Oliveira QC, Santos RA Marques MD, Forgiarini Júnior LA. Existe associação entre massa e força muscular esquelética em idosos hospitalizados? Rev Bras Geriatr Gerontol. 2016;19(2):257-264.

27. Araújo APS, Bertolini SMMG, Martins Junior J. Alterações morfofisiológicas decorrentes do processo de envelhecimento do sistema musculoesquelético e suas consequências para o organismo humano. Persp Online: Biol \& Saúde. 2014; 12(4):22-34.

28. Maslow AL, Sui X, Colabianchi N, Hussey J, Blair SN. Muscular strength and incident hypertension in normotensive and prehypertensive men. Med Sci Sports Exerc. 2010; 42(2):288-295.

29. Heyward VH, Gibson AL. Advanced fitness assessment exercise prescription. 7a ed. Champaign: Human Kinetics; 2014.

30. Figeuiredo IM, Sampaio RF, Mancini MC, Silva FCM, Souza MAP. Teste de força de preensão utilizando o dinamômetro Jamar. Acta fisiatr. 2007; 14(2):104-110.31. Vieira MCA, Souza CF, Câmara SMA, Matos GSR, Moreira MA, Maciel ACC. Relação entre força de preensão manual e força de membro inferior em mulheres de meia idade: umestudo transversal. Rev Bras Ativ Fis Saúde. 2015; 20(5):467-475.

32. Oliveira AGP, Oliveira TP, Ferreira MEC, Lima JRP. Diferença entre peso e estatura auto-referidos e aferidos para o cálculo do indice de massa corporal e sua relação com a imagem corporal. HU Rev. 2008; 34(3):179-183. 